\title{
Enhanced Event Triggered Localization Algorithm with a Parameter of Energy Transmission and Energy Received in Mobile Wireless Sensors Networks
}

\author{
Preeti Singh \\ M.Tech Student (ECE), \\ Indo Global College of Engineering (PTU) Mohali, \\ Punjab-140109, INDIA
}

\author{
Hardeep Singh Saini, Ph.D \\ Professor (ECE) and Associate Dean \\ Academic, Indo Global College of Engineering \\ (PTU) Mohali, Punjab-140109, IIA
}

\begin{abstract}
Many localization algorithm proposed for wireless sensor networks(WSN) .Localization is a term that can be defined as the determination of the exact physical location of each sensor node in a geographic map or to find its relative location from a topology map in a given network structure. Many localization algorithms for MWSN are less accurate and more time consuming. Localization In this paper, first, we propose a localization algorithm in which distance is estimated from sink node to person and GPS to person. Second, we transmit energy of sink node to person and transmit energy of GPS to person.
\end{abstract}

\section{Keywords}

MWSN, GPS, WSN.

\section{INTRODUCTION}

Wireless sensor networks built up of sensor nodes which consist of sensing, computing, communication, actuation and power components that cooperatively perform the task of collecting relevant data and monitor its surrounding for some change or event to occur. WSN's has its own features that not only differentiate it from other wireless networks. In WSNs, sensor nodes are deployed in different locations to be responsible for perceiving local information and reporting to sink nodes. Sink nodes are distinguished devices with powerful computation capacity, large memory size , high power energy and long communication range, so as to collect data from SNs. Sink nodes act as the gateway between WSNs and the end user.

The original idea to localize mobile sensor nodes is using a global positioning system (GPS) on every mobile sensor. But considering the cost and power consumption, the GPS solution is not acceptable in many applications. Some special sensor nodes which used namely called as anchor nodes which already know their absolute Locations via GPS or manual placement. Other sensor nodes estimate their locations based on the information provided by these anchor nodes.

In this paper we first estimate the distance and then transmit energy of sink node to person and transmit energy of GPS to person in the form of graph

The rest of the paper organized as follows. Section II provides an overview of existing localization algorithm using anchor nodes. In section III, our localization scheme is proposed. The simulation and analysis are presented in section IV. In section $\mathrm{V}$ we draw our conclusion.

\section{RELATED WORK}

A Large number of existing localization schemes have been proposed to solve the localization problems in WSNs. Each sensor nodes uses a technique or several techniques in order to calculate distance. Literature [1] provides detailed survey of secure and energy efficient adaptive routing for wireless sensor networks. This paper proposed a secure and energy efficient adaptive routing (SEEAR) protocol for wireless sensor networks, which is secure and energy efficient. To reduce energy, SEEAR establishes communication link between two nodes, only if the distance between these nodes is less than some threshold value and they satisfied the key criteria of Q composite keys. Literature [2] provides detailed survey of accurate localization technique [ALT]. ALT provides a pin point location information to the sensor nodes without any expense of cost or power. It also introduces the concept of location aware IDs for SNs. Ssu and Guo proposed a localization algorithm using a mobile anchor node. In their algorithm, a mobile anchor node moves around in a sensor area and periodically broadcasts beacon messages which include its current location information. A stationary sensor node receives and records the first and last locations of the mobile anchor node when its move into the communication ranges of the stationary sensor node. Literature [3] presents an energy efficient localization algorithm for wireless sensor networks using a mobile anchor node. It is based on the distance measurement with extra hardware. The mobile node is equipped with a GPS receiver, RF (radio frequency) and ultrasonic transmitter. Each stationary sensor node is equipped with a RF and ultrasonic receiver. The mobile nodes periodically broadcast its location information and stationary sensor nodes take the current position of the mobile node as a virtual anchor point. The location of a sensor node is computed by measuring the distance to the virtual anchor point using TDOA (time difference of arrival) method.

Although many protocols and algorithms have been proposed for WSNs in recent years, they may not be suitable to MWSNs. Based on IR fingerprinting and RSSI technique, we propose an enhanced triggered algorithm with a parameter of energy transmission and energy received in MWSN.

\section{ALGORITHM DESIGN}

In this section we introduce a localization algorithm for mobile sensor network. It is based on equation (1) and (2) represent the energy dissipation, when a $\mathrm{SN}$ sends or receives an 1 bit message

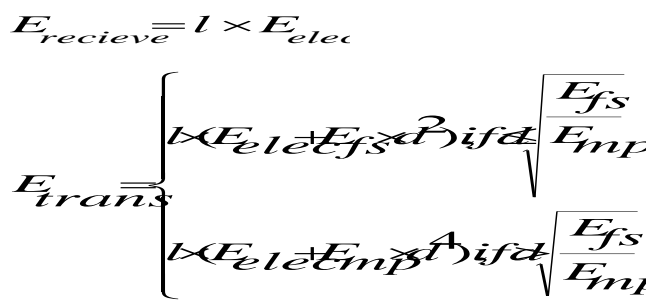

(1) 
1 is the size of message i.e. packet size and taken it 64 and $d$ is the distance. Rest of the parameters is same. And ignore the ifd part. Put the values up to $d^{2}$ and $d^{4}$ only. Graph will show the variations.

\subsection{Simulation Parameters}

- The field dimensions $\mathrm{x}$ and $\mathrm{y}$ in meters is taken as $100 \mathrm{~m}$

- $\quad \mathrm{X}$ and $\mathrm{Y}$ co- ordinates of the sink is 0.5

- Free space attenuation coefficient $\left(\mathrm{E}_{\mathrm{fs}}\right)$ is $10 \mathrm{pJ} / \mathrm{bit} / \mathrm{m}^{2}$.

- Multipath attenuation coefficient $\left(\mathrm{E}_{\mathrm{mp}}\right)$ is 0.0013 $\mathrm{pJ} / \mathrm{bit} / \mathrm{m}^{4}$.

- Electronic power $\left(\mathrm{E}_{\text {elec }}\right)$ is $50 \mathrm{~nJ} / \mathrm{bit}$.

1. Distance Estimation

Distance is estimated with the formulae is given by

- SINK NODE TO PERSON

$\mathrm{di}=\sqrt{ }(\mathrm{x}-\mathrm{xi})^{2}-\left(\mathrm{y}-\mathrm{y}_{\mathrm{i}}\right)^{2}$

- GPS TO PERSON

$$
\mathrm{d} 1==\sqrt{ }(\mathrm{x}-\mathrm{xi})^{2}-\left(\mathrm{y}-\mathrm{y}_{\mathrm{i}}\right)^{2} \text { GPS TO PERSON }
$$

2. Transmit Energy Of Sink Node To Person Is Given By

$$
\text { Etrans } 2=1 *\left(\text { Elec }+ \text { Emp } * d^{4}\right.
$$

3. Transmit Energy Of Gps To Person Is Given By Etrans $21=1 *\left(\right.$ Elec+Emp*d $\left.1^{4}\right)$

\subsection{DISTANCE ESTIMATION}

Distance is estimated from a sink node to person. fig shows person starts moving from 0 and distance of person to floor is $100 \mathrm{~m}$.

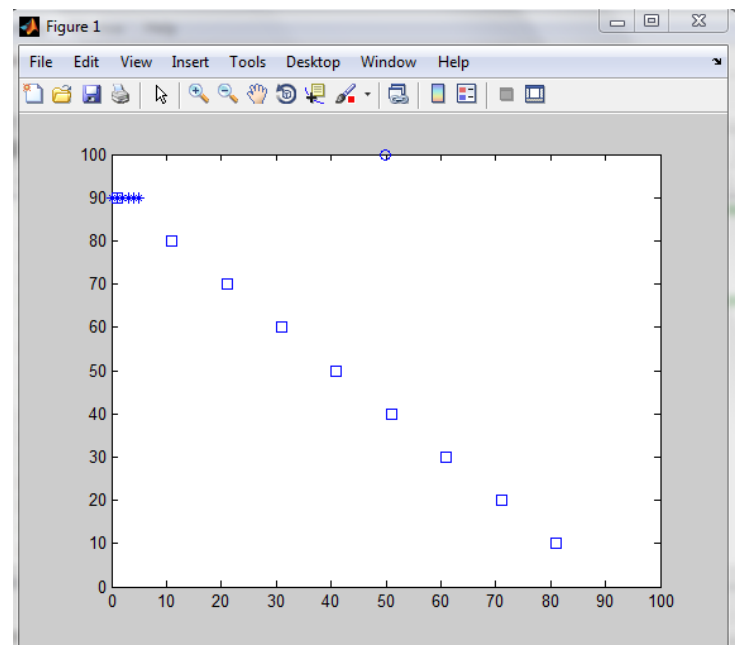

Total distance estimated is $100 \mathrm{~m}$

Graph of transmit energy of sink node to person is plot between sink energy and range in meters

\section{RESULTS}

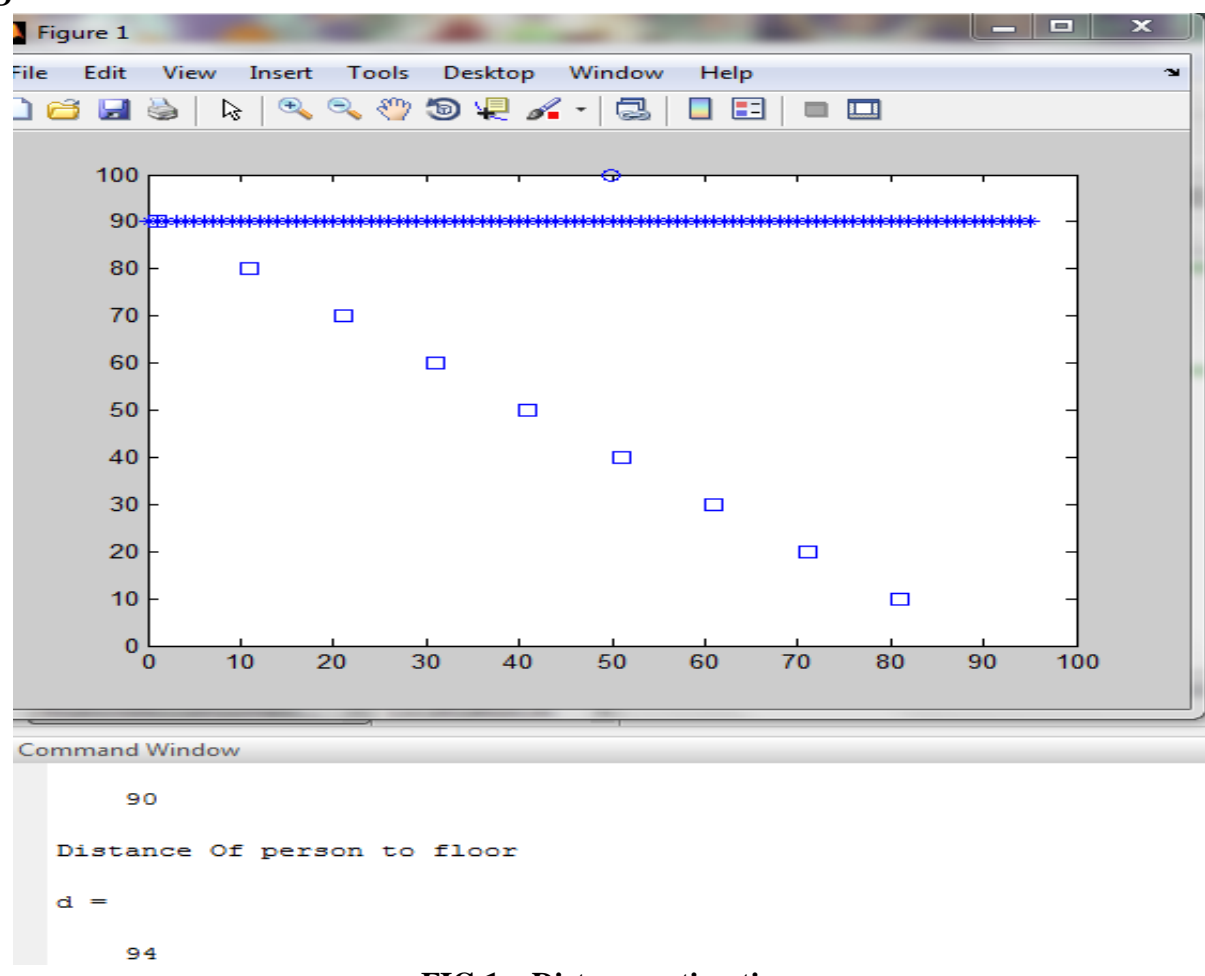

FIG 1 - Distance estimation

\subsection{ENERGY TRANSMISSION AND ENERGY RECIEVED}


Graph of transmit energy of GPS to person is plot between GPS energy vs range in meters

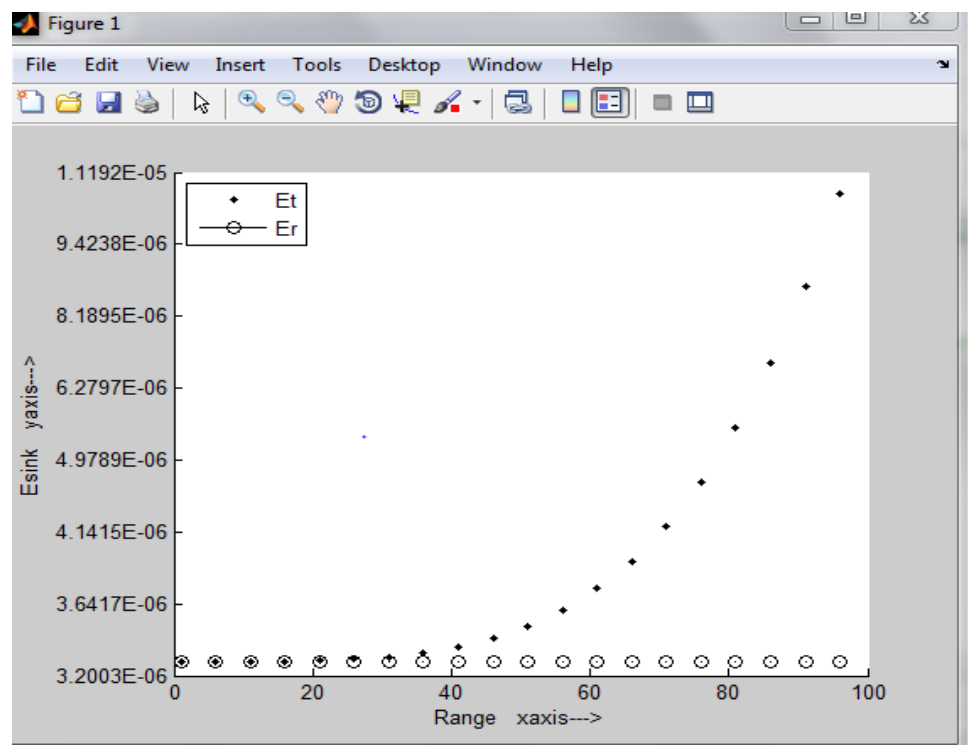

FIG 2 - Energy Transmission

here Et is transmitted energy

Er is received energy

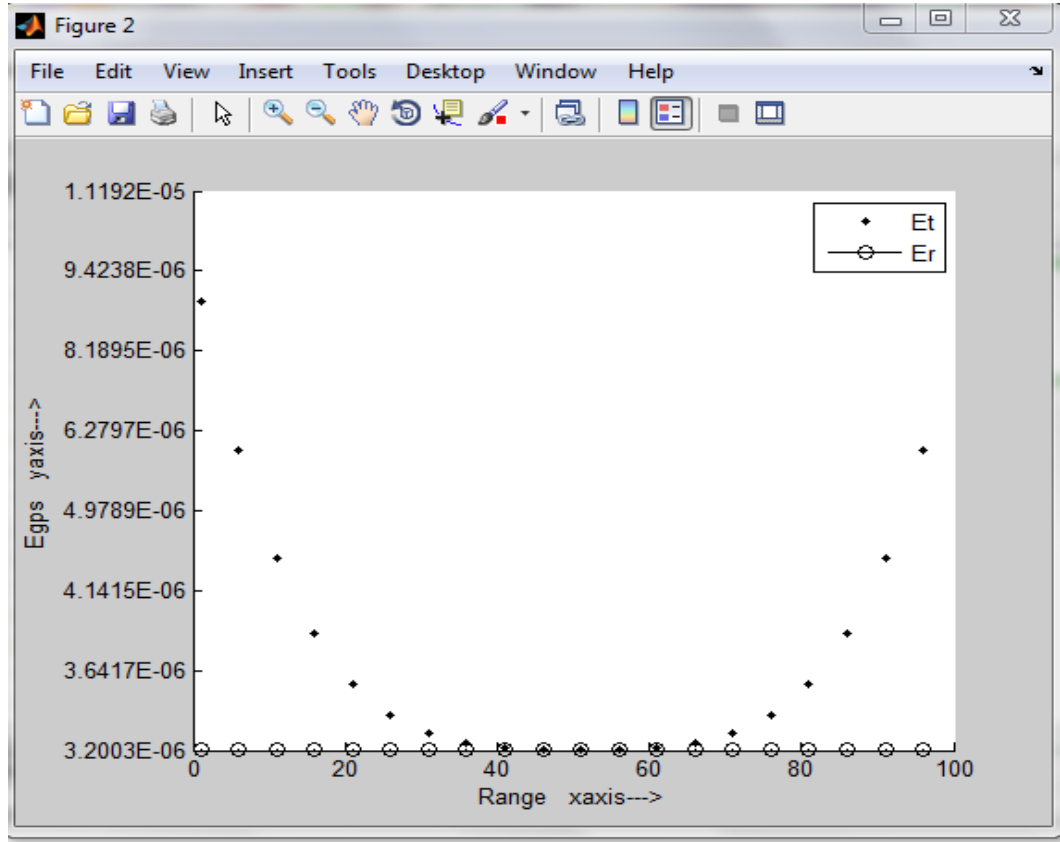

FIG 3 - ENERGY RECIEVED

These three are the results of this paper

First result is distance estimation of person to the floor

Second result is transmit energy of sink node to the person

Third result is transmit energy of GPS to the person

1 is the size of message i.e. packet size and taken it 64 and $d$ is the distance. Rest of the parameters is same. And ignore the ifd part. Put the values up to $d^{2}$ and $d^{4}$ only. Graph will show the variations. 


\section{SIMULATION}

In this section, we discuss the simulation of our proposed localization algorithm. MATLAB with coding of WSN is adopted in the simulation.

Simulation environment

In our simulations, the sensor area is $100 \times 100 \mathrm{~m}^{2}$. The anchor nodes manually deployed in the area, which is equipped with GPS to obtain their

position. The mobile sensor node moves in the sensor area in any directional

\section{CONCLUSION}

Many wireless sensor networks require locations of sensor nodes accurately. Locating a SN in a WSN is required in most of the applications. In this paper first movement of the person on a particular floor is shown from intial position to final position as shown above in graph. Secondly, In next graph sink node energy and GPS energy is shown at a particular range this parameter is also shown above in a form of graph.

\section{REFERENCES}

[1] D Prasad . Manik Gupta , Dr R.B Patel "SEEAR:secure and energy efficient adaptive routing for wireless sensor networks" In

[2] Ajay Kr. Gautam , Amit Kr. Gautam "Accurate Localization Technique using virtual coordinate system in wireless sensor networks" IJRTE vol 2, No. 5, November 2009

[3]B. Zhang and F.Yu " An energy efficient algorithm for wireless sensor networks using a mobile anchor node" International conference on information and automation , June 2008

[4] M. B. Srivastava , R. Muntz and M. Potkonjak, "Smart Kindergarten: Sensor-based Wireless Networks for Smart Developmental Problem-solving Environments", In Proceedings of Seventh annual International Conference on Mobile Computing and Networking, July 2001, Rome, Italy, pp. 132-138.

[5] Y. Shang, W. Ruml, Y. Zhang, and M. Fromherz, "Localization from mere connectivity", In Proceedings of ACM Symposium on Mobile Ad Hoc Networking and Computing (MobiHoc'03), June 2003, Annapolis, Maryland, USA, pp. 201-212.

[6] Anushiya A Kannan, Guoqiang Mao and Branka Vucetic, "Simulated Annealing based Wireless Sensor Network Localization", Journal of Computers, Vol. 1, No. 2, pp 15-22, May 2006.

[7] Cesare Alippi, Giovanni Vanini, "A RSSI-based and calibrated centralized localization technique for Wireless Sensor Networks", in Proceedings of Fourth IEEE International Conference on Pervasive Computing and Communications Workshops (PERCOMW'06), Pisa, Italy, March 2006, pp. 301-305.

[8] T. He, C. Huang, B. Blum, J. Stankovic, and T. Abdelzaher, "Range-free localization schemes in large scale sensor networks", In Proceedings of the Ninth Annual International Conference on Mobile Computing and Networking (MobiCom'03), September 2003, San Diego, CA, USA, pp. 81-95.

[9] A. Savvides, H. Park, and M. Srivastava, "The bits and flops of the n-hop multilateration primitive for node localization problems", In Proceedings of the 1st ACM international Workshop on Wireless Sensor Networks and Applications (WSNA'02), September 2002, Atlanta, Georgia, USA, pp. 112-121.

[10] S. Simic and S. Sastry, "Distributed localization in wireless ad hoc networks", Technical Report UCB/ERL M02/26, UC Berkeley, 2002, Available HTTP.

[11] J. Bachrach, R. Nagpal, M. Salib and H. Shrobe, "Experimental Results for and TheoriticalAnalysis of a Self-Organizing a Global Coordinate System from Ad Hoc Sensor Networks", Telecommunications System Journal, Vol. 26, No. 2-4, pp. 213-233, June 2004

[12] N. Priyantha, H. Balakrishnan, E. Demaine, and S. Teller, "Anchor-free distributed localization in sensor networks", MIT Laboratory for Computer Science, Technical Report TR-892, April 2003.

[13] C. Savarese, J. Rabaey, and J. Beutel, "Locationing in distributed ad-hoc wireless sensor networks", in Proceedings of IEEE International Conference on Acoustics, Speech, and Signal Processing (ICASSP'01), May 2001, Salt Lake City, Utah, USA, vol. 4, pp. 2037 2040.

[14] David Moore, John Leonard, Daniela Rus, and Seth Teller, "Robust distributed network localization with noisy range measurements", in Proceedings of the Second ACM Conference on Embedded Networked Sensor Systems (SenSys'04), November 2004, Baltimore, MD, pp. 50-61.

[15] Lambert Meertens and Stephen Fitzpatrick, "The Distributed Construction of a Global Coordinate System in a Network of Static Computational Nodes from InterNode Distances", Kestrel Institute Technical Report KES.U.04.04, Kestrel Institute, Palo Alto, 2004, Available FT

\section{AUTHOR PROFILE}

Er. Preeti Singh was born in 1988. She is M. Tech student of ECEdepartment since 2011at Indo Global College ofEngineering and thesis working on her M. Tech thesis. She is studying Mobile wireless sensor networks and their algorithms, characteristics and applications.

Dr. Hardeep Singh Saini obtained his Doctorate degree in Electronics andCommunication Engineering in $2012 . \mathrm{He}$ holds Master's degree in Electronics and Communication Engineering from Punjab Technical University, Jalandhar passed in 2007. His total experience is 14 years, presently working as Professor (ECE) and Associate Dean Academic at Indo Global College of Engineering, Abhipur (Mohali), and PUNJAB (INDIA) since June-2007.He is author of 5 books in the field of Communication Engineering. He has presented 21 papers in international/national conferences and published 23 papers in international journals. He is a life member of the IETE (India). 